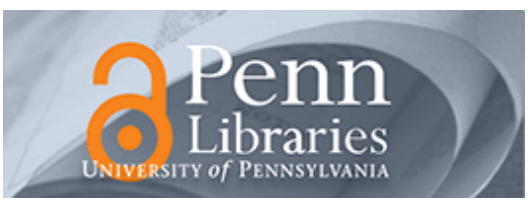

University of Pennsylvania

ScholarlyCommons

6-27-2007

\title{
Comparing Flow Thresholds and Dynamics for Oscillating and Inclined Granular Layers
}

\author{
Sebastien Aumaitre \\ Haverford College \\ Conor Puls \\ Haverford College \\ Jim N. McElwaine \\ University of Cambridge \\ Jerry P. Gollub \\ Haverford College; University of Pennsylvania, jgollub@sas.upenn.edu
}

Follow this and additional works at: https://repository.upenn.edu/physics_papers

Part of the Physics Commons

\section{Recommended Citation}

Aumaitre, S., Puls, C., McElwaine, J. N., \& Gollub, J. P. (2007). Comparing Flow Thresholds and Dynamics for Oscillating and Inclined Granular Layers. Retrieved from https://repository.upenn.edu/physics_papers/ 144

Suggested Citation:

S. Aumaitre, C. Puls, J.N. McElwaine and J.P. Gollub. (2007). Comparing flow thresholds and dynamics for oscillating and inclined granular layers. Physical Review E 75, 061307.

(C) 2007 The American Physical Society

http://dx.doi.org/10.1103/PhysRevE.75.061307

This paper is posted at ScholarlyCommons. https://repository.upenn.edu/physics_papers/144

For more information, please contact repository@pobox.upenn.edu. 


\title{
Comparing Flow Thresholds and Dynamics for Oscillating and Inclined Granular Layers
}

\begin{abstract}
The onset and dynamics of flow in shallow horizontally oscillating granular layers are studied as a function of the depth of the layer and imposed acceleration. Measurements of the flow velocity made from the top and side are presented in the frame of reference of the container. As is also found for avalanches of inclined layers, the thresholds for starting and stopping of flow are slightly different. The variation with depth of the starting acceleration $\Gamma_{\text {start }}$ for the oscillating layer is similar to the corresponding variation of the tangent of the starting angle $\tan \left(\Theta_{\text {start }}\right)$ for avalanches in the same container at low frequencies, but deviates as the frequency is increased. However, the threshold behavior depends significantly on the measurement protocol. Just above $\Gamma$ start, the motion decays with time as the material reorganizes over a minute or so, causing the apparent threshold to increase. Furthermore, the rms velocity as a function of acceleration rises more sharply above the starting threshold if the first minute or so of excitation is discarded. Once excited, the rheology of the material is found to vary in time during the cycle in surprising ways. If the maximum inertial force (proportional to the container acceleration amplitude) is slightly higher than that required to produce flow, the flow velocity grows as soon as the inertial force exceeds zero in each cycle, but jamming occurs long before the inertial force returns to zero. At higher $\Gamma$, the motion is fluidlike over the entire cycle. However, the fraction of the cycle during which the layer is mobile is typically far higher than what one would predict from static considerations or the behavior of the inclined layer. Finally, we consider the flow profiles as a function of both the transverse distance across the cell at the free surface and also as a function of the vertical coordinate in the boundary layer near the sidewall. These profiles have time-dependent shapes and are therefore significantly different from profiles previously measured for avalanche flows.
\end{abstract}

\section{Disciplines}

Physical Sciences and Mathematics | Physics

\section{Comments}

Suggested Citation:

S. Aumaitre, C. Puls, J.N. McElwaine and J.P. Gollub. (2007). Comparing flow thresholds and dynamics for oscillating and inclined granular layers. Physical Review E 75, 061307.

(C) 2007 The American Physical Society

http://dx.doi.org/10.1103/PhysRevE.75.061307 


\title{
Comparing flow thresholds and dynamics for oscillating and inclined granular layers
}

\author{
S. Aumaitre, ${ }^{1}$ C. Puls, ${ }^{1}$ J. N. McElwaine, ${ }^{2}$ and J. P. Gollub ${ }^{1,3}$ \\ ${ }^{1}$ Physics Department, Haverford College, Haverford, Pennsylvania 19041, USA \\ ${ }^{2}$ Department of Applied Mathematics and Theoretical Physics, University of Cambridge, Cambridge CB2 1TN, United Kingdom \\ ${ }^{3}$ Physics Department, University of Pennsylvania, Philadelphia, Pennsylvania 19104, USA
}

(Received 30 September 2006; published 27 June 2007)

\begin{abstract}
The onset and dynamics of flow in shallow horizontally oscillating granular layers are studied as a function of the depth of the layer and imposed acceleration. Measurements of the flow velocity made from the top and side are presented in the frame of reference of the container. As is also found for avalanches of inclined layers, the thresholds for starting and stopping of flow are slightly different. The variation with depth of the starting acceleration $\Gamma_{\text {start }}$ for the oscillating layer is similar to the corresponding variation of the tangent of the starting angle $\tan \left(\theta_{\text {start }}\right)$ for avalanches in the same container at low frequencies, but deviates as the frequency is increased. However, the threshold behavior depends significantly on the measurement protocol. Just above $\Gamma_{\text {start }}$, the motion decays with time as the material reorganizes over a minute or so, causing the apparent threshold to increase. Furthermore, the rms velocity as a function of acceleration rises more sharply above the starting threshold if the first minute or so of excitation is discarded. Once excited, the rheology of the material is found to vary in time during the cycle in surprising ways. If the maximum inertial force (proportional to the container acceleration amplitude) is slightly higher than that required to produce flow, the flow velocity grows as soon as the inertial force exceeds zero in each cycle, but jamming occurs long before the inertial force returns to zero. At higher $\Gamma$, the motion is fluidlike over the entire cycle. However, the fraction of the cycle during which the layer is mobile is typically far higher than what one would predict from static considerations or the behavior of the inclined layer. Finally, we consider the flow profiles as a function of both the transverse distance across the cell at the free surface and also as a function of the vertical coordinate in the boundary layer near the sidewall. These profiles have time-dependent shapes and are therefore significantly different from profiles previously measured for avalanche flows.
\end{abstract}

DOI: 10.1103/PhysRevE.75.061307

PACS number(s): 45.70.Ht, 83.80.Fg

\section{INTRODUCTION}

The onset of granular avalanches on an inclined plane has been widely studied in numerical and laboratory experiments $[1-5]$ in part because of their geophysical significance. Although various configurations have been studied, including also heap flows and rotating drums with various material properties, some common features have been noted for these dense surface flows. When the surface is inclined to a critical angle $\theta_{\text {start }}$, flow begins, and when the angle is subsequently reduced to a somewhat lower angle, flow stops. In the case of the inclined plane with a rough bottom surface, the critical angles depend on the layer depth. A single phenomenological law has been proposed to describe the reduction of the critical angles when the depth of the layer is increased. A major theoretical challenge is to obtain a complete model that can capture all the dynamical properties from the solidlike state below the critical angle to the completely fluidized state far above this angle [6-10]. Recently, it has been shown that for fully developed channel or avalanche flows of dense granular matter, the depth dependence of the velocity profile and the effects of no-slip walls can be described by means of a single constitutive law for the rheology of dense granular flow [11-15]. However this rheology only holds for deep flows where the velocity profile is a power law and the shear rate on the free surface is zero. For shallow flows, the vertical profile becomes almost linear and the shear rate does not vanish on the surface $[4,13]$.
The transition from static compact to mobile granular layers can be also studied by subjecting a container to a time periodic horizontal displacement of the form

$$
x(t)=A \sin \omega t,
$$

where $A$ is the amplitude and $\omega$ is the angular frequency. The oscillatory and avalanche experiments can be related to each other if a reference frame at rest with respect the container is used. Then, the fictitious force per unit mass acting on the particles in the oscillating container's frame of reference is the negative of the acceleration, i.e., $A \omega^{2} \sin \omega t$. The ratio of the maximum of this fictitious force to the layer weight, $\Gamma$ $=A \omega^{2} / g$, is proportional to the ratio of the shear stress to the normal stress, which must be sufficiently large to overcome static friction in order to initiate the motion of the grains relative to the container. In the steady avalanche experiment, this ratio is given by $\tan \theta$, where $\theta$ is the inclination angle of the plane. Therefore, the avalanche experiment might be regarded provisionally as the low frequency limit of the oscillating flow. At finite frequency, the two systems would be different, but the forcing frequency can be useful in probing the rheology. A difficulty of the avalanche experiments is that there are only two control parameters, $h$ and $\theta$, which are closely related for steady flows. Oscillatory excitation introduces a new time scale, and generates periodic flows for all parameter choices. This enables a wide range of flows to be studied, and allows good statistics to be collected by averaging over multiple cycles. 
Earlier experimental studies of horizontally oscillating layers have considered the fluid-solid phase transition in a single layer of grains when the filling fraction and the driving strength are increased $[16,17]$. Two-dimensional segregation phenomena have also been studied under oscillatory excitation [18]. In deeper layers, a slow convective motion was discovered and studied $[19,20]$. This secondary flow is due to the lateral walls, where a downward stream is generated. In a more complete study $[15,21]$, the onset of the grain motion was measured as a function of layer depth in a relatively narrow cell $(\sim 30$ particle diameters wide), with rough particles and a smooth bottom surface. In this previous work, in contrast to the behavior to be reported here, the starting and stopping accelerations increase with the layer depth. We return to this issue in the concluding section.

In the present study we consider the thresholds $\Gamma_{\text {start }}$ and $\Gamma_{\text {stop }}$ for motion relative to the container under oscillatory excitation, and we compare the results to the critical angles for inclined flows in the same container. (In these studies, the particles are rough, as is the bottom surface of the container.) Clear thresholds are found, and their dependence on layer depth is similar to the avalanche case at low frequencies. However, the threshold behavior depends substantially on the measurement protocol, due to particle rearrangements that occur over the first minute or so of excitation. Changes in rheology due to particle rearrangements have been noted in shear flows where there are changes in shear direction [22], and the effects we note here could be related. Furthermore, the time-dependence of the flow behavior within the cycle is surprising. In particular, the motion is found to be fluidlike during part of the cycle and solidlike during the remainder of the cycle. Strikingly, the mobile interval is longer than one would predict from the acceleration and the measured thresholds. Finally, we consider the variation of the velocity with distance across the cell at the top, and vertically within the boundary layer. We show that these profiles are inherently time-dependent, and usually different from those found for steady flows.

\section{EXPERIMENTAL METHODS}

A sketch of the experimental device is shown Fig. 1. The experiments are performed with polydisperse glass spheres with diameters between $0.71 \mathrm{~mm}$ and $1.20 \mathrm{~mm}$ and a mean diameter $d=0.96 \mathrm{~mm}$. The grains are contained in a Plexiglass cell of length $L=200 \mathrm{~mm}$, width $W=80 \mathrm{~mm}$, and height $H=60 \mathrm{~mm}$. The height of the layer $h$ is deduced from the volume of grains placed in the container. The range in $h$ was 1 to $16 d$ with aspect ratios $h / W$ (or $h / L$ ) varying from 0.01 to 0.2 (or 0.004 to 0.08 ). The rough bottom of the container is made of grains glued to the surface. The cell is oscillated horizontally by an electro-magnetic shaker (VTS model 40c) controlled by a function generator (HP 3325) and amplifier (Techron5507).

Several different arrangements for coupling the cell to the shaker were used. One method, shown in Fig. 1(a), relies on a linear roll bearing and a steel shaft. In a second approach, the cell rests on a one-dimensional supported track that prevents gravitational torques from being applied to the shaft,

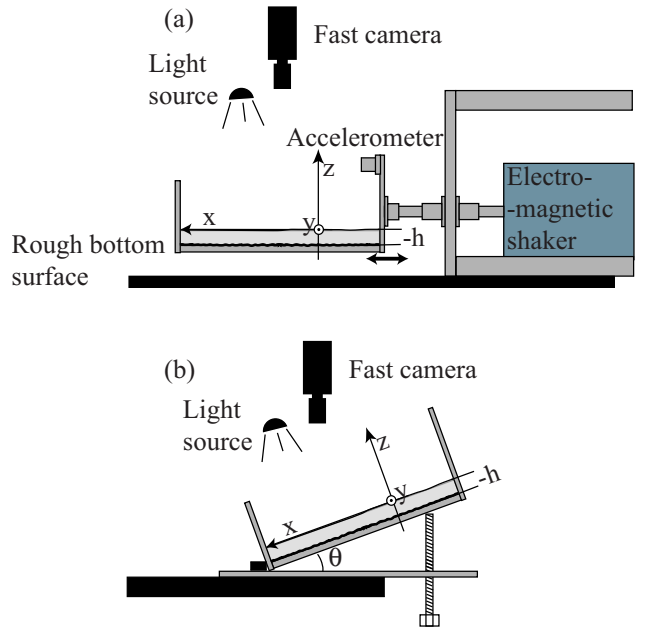

FIG. 1. (Color online) (a) Sketch of the horizontally oscillated experiment. A plexiglass cell containing the granular layer is attached to an electromagnetic shaker via a steel shaft confined by a low friction roll-bearing. A CCD camera is used to track the grain motion from the top and the side. (b) Sketch of the avalanche experiment performed in the same cell with the inclination angle $\theta$. The coordinate axes are shown, with motion always along $x$.

and also avoids enhanced friction at the instant of zero velocity. The resulting time-dependent acceleration in this second case is closer to a sinusoid.

The range of oscillation frequencies $f_{0}=\omega / 2 \pi=1 / T$ used in this experiment extends from 3 to $10 \mathrm{~Hz}$. The cell acceleration is measured by an accelerometer (Dytran 3134A). A Kodak SR-500 MotionCorder camera is used to record the particle positions; the frame size is 512 by 240 pixels. Measurements are made from both the top and side of the container. The thresholds and time variations of spatially averaged surface velocities near the center of the cell, in an area of 30 by 15 grain diameters (or 15 by 15 for the highest excitation frequencies), by means of particle-tracking from the top. The horizontal velocity profile must be determined over a somewhat larger region (58 by $27 d$ starting from the sidewall) that is at least half the width of the cell. For measurements made from the side, the width of the picture is adjusted to be slightly larger than the layer depth. A frame rate of $500 \mathrm{~Hz}$ is generally used (or $1000 \mathrm{~Hz}$ for the highest excitation frequencies).

Despite the use of polydisperse particles to suppress ordering, we find evidence for particle rearrangements over times as short as $10 \mathrm{sec}$. This effect causes apparent thresholds to evolve over a minute or so of excitation. Therefore, we choose to make threshold measurements in two different ways. First, we made "fast" measurements (method A), where the velocity measurements are performed a few cycles after the prescribed amplitude is applied. To measure $\Gamma_{\text {start }}$, we start with the material at rest and increase the amplitude to the measurement acceleration. To measure $\Gamma_{\text {stop }}$, the granular bed is first driven for few cycles at high amplitude where the flow is continuous, and then the amplitude is reduced to the target acceleration, where the velocities are measured (a few cycles after the decrease). The material is refreshed by stirring each time the layer depth is increased. 

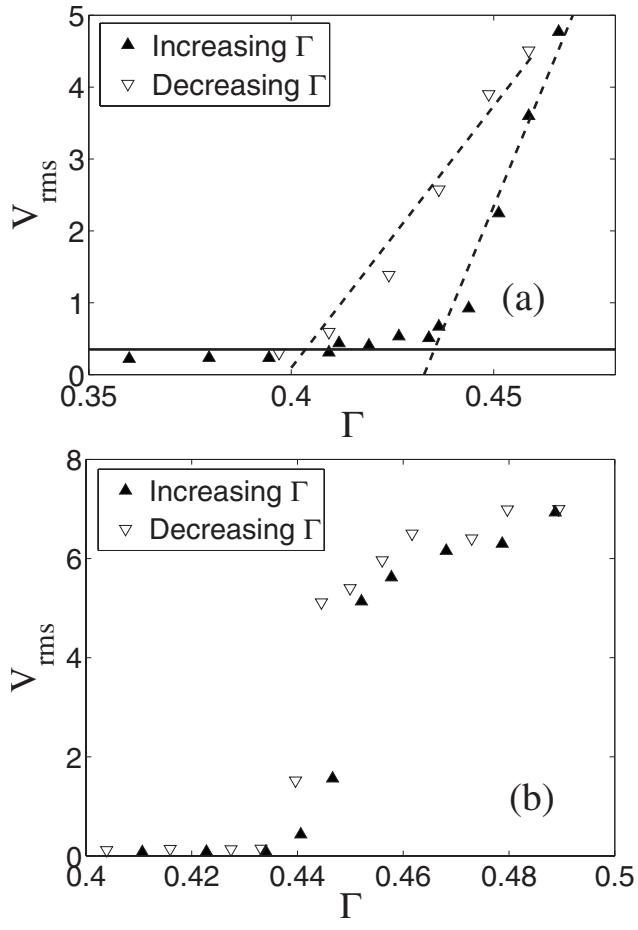

FIG. 2. Acceleration dependence of the rms $x$ velocity at the free surface of the grain layer for depth of $7 d$, as a function of the dimensionless acceleration $\Gamma$. All velocity measurements in this paper are in the box frame of reference. The solid upward pointing triangles are obtained when the acceleration is increased. The open downward pointing triangles are obtained when the acceleration is decreased. (a) Method A (see text). Dashed lines show the best linear fit to the rms velocity emerging from the background noise (full line). (b) Method B, in which measurements are made after the transient decay $(1 \mathrm{~min})$. Here, the rise is sharper, and the hysteresis less. In both cases the nondimensional frequency $\eta=0.21\left(f_{o}\right.$ $=3.3 \mathrm{~Hz}$ ).

We also used a second protocol (method B), where the material is oscillated for $1 \mathrm{~min}$ at the prescribed amplitude (instead of few cycles) before starting the velocity measurement. In this case the material is stirred after each measurement to obtain similar initial conditions.

Surface particles which reflect light from an extended source placed close to the camera axis are tracked using IDL software [23]. The particle motion is determined relative to the container, using a marker at rest with respect to the container. For measurements made from the side, there is some damping of motion due to boundary friction, but we are still able to determine the depth dependence adequately. All velocity measurements in this paper are presented in the box frame of reference. In this non-inertial frame, the flow is driven by time-dependent effective body forces, and retarded by boundary friction.

In most of our experiments we vary $\Gamma$ by changing the amplitude $A$ and then compare the behavior at different oscillation frequencies. We express our experimental results as function of the following dimensionless parameters: the dimensionless acceleration, $\Gamma=A \omega^{2} / g$, the height of the layer normalized by the grain diameter, $h / d$, and the dimensionless frequency, $\eta=\omega \sqrt{d / g}$. Notice that the limit $\eta \rightarrow 0$ with
$\Gamma$ constant corresponds to the avalanche case with slope $\tan ^{-1} \Gamma$. Our oscillating experiments are performed in a range: $0.18<\eta<0.62$. We choose to normalize the velocity by $f_{o} d$, where $f_{0}=\omega / 2 \pi$, in order to have a dimensionless velocity smaller than 1 for particles moving less than one diameter during one oscillation period, $T$. Conventions for coordinates are given in Fig. 1; $x$ is the direction of motion and $z$ is vertical.

\section{RESULTS: THRESHOLDS}

Using particle tracking data, we compute the rms (rootmean-square) $x$ velocity $V_{\text {rms }}$ of the grains, averaged in space and time, as a function of the dimensionless acceleration $\Gamma$. Figure 2(a) shows these curves for a layer of thickness $h$ $=7 d$ when the acceleration is increased (upward solid triangles) and then decreased (downward open triangles) using method A (Sec. II), where the excitation time is too short to allow ordering to develop. Both curves show a clear threshold where the rms velocity starts to emerge from a noisy background, but this threshold is clearly smaller when the acceleration is reduced. We define the thresholds using linear extrapolation. Note that if the maximum velocity over the cycle is used instead of the rms value, similar results are obtained.

The threshold accelerations are also measured using method B (Sec. II), where the first minute of data is discarded for each point in order to allow the material to reach a steady state. In this case, the resulting velocity as a function of $\Gamma$ [Fig. 2(b)] rises more sharply (and not linearly) above the threshold. Also, the degree of hysteresis is less than with method A.

If $\Gamma$ is increased in a discrete step (which need not be large) to a value in the transition region, motion is induced, but it can then decay after a few seconds or tens of seconds, as shown in Fig. 3. If the acceleration is sufficiently large, there is no decay, but in the transition region, the decay can

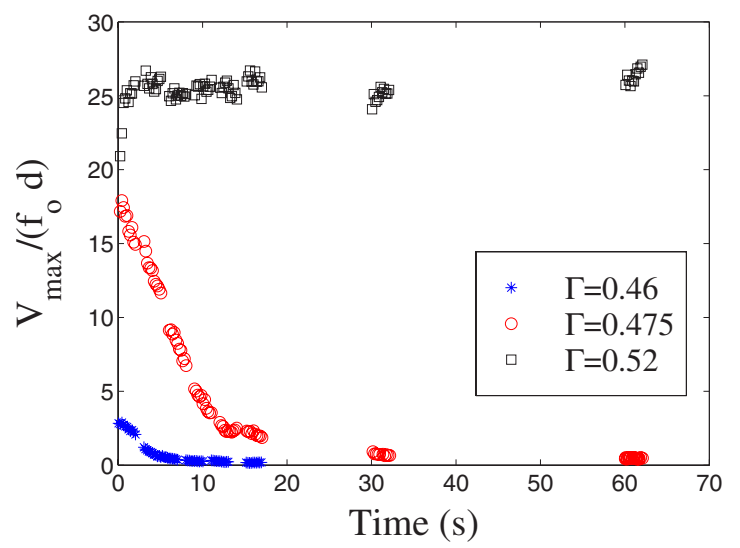

FIG. 3. (Color online) Transient decay of the surface $x$-velocity amplitude (or maximum surface velocity) for three accelerations near threshold, as a result of rapid reorganization of the grains for $\eta=0.31\left(f_{o}=5.0 \mathrm{~Hz}\right)$. Velocities are normalized by $f_{o} d$, so the displacements are many particle diameters. For sufficiently large $\Gamma$, no decay is observed. In method $\mathrm{B}$, thresholds are determined after the decay (if present). 

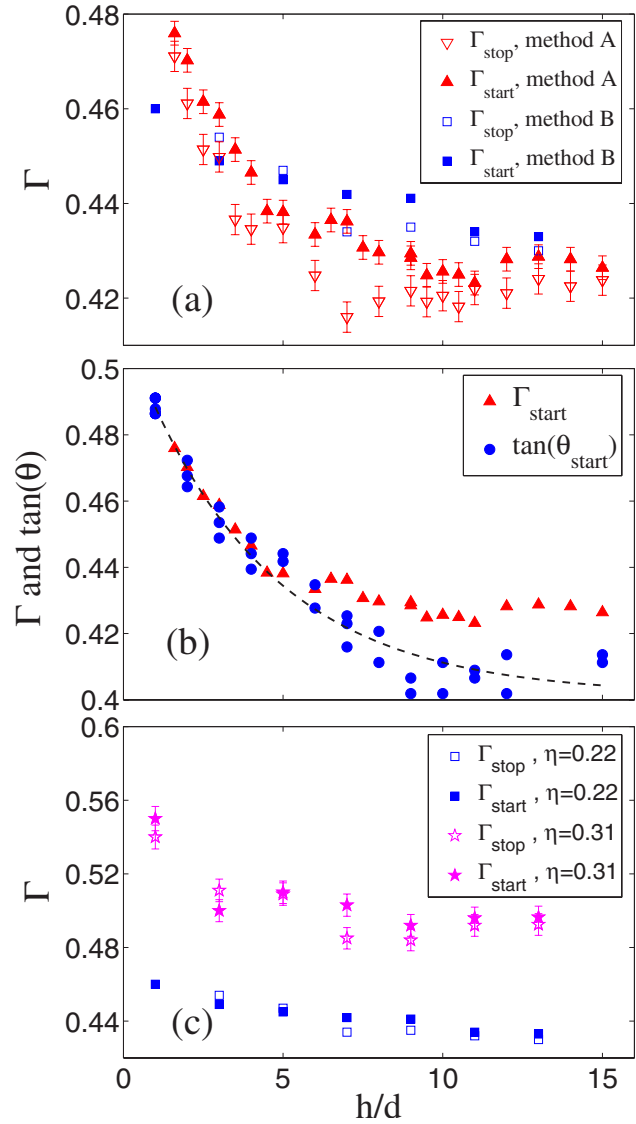

FIG. 4. (Color online) Dependence of the thresholds $\Gamma_{\text {start }}$ and $\Gamma_{\text {stop }}$ on the layer depth. In all cases, solid symbols denote upward transitions and open symbols denote downward transitions. (a) Comparison of the two experimental procedures, for excitation at $\eta=0.22\left(f_{o}=3.5 \mathrm{~Hz}\right.$; triangles, method A; squares, method $\left.\mathrm{B}\right)$. (b) Comparison of the oscillating layer to the avalanche experiment. Triangles: Oscillating driving (method A, $\eta=0.22$ ). Circles: Tangent of the critical avalanche inclination angle $\theta_{\text {start }}$. The dashed line is the fit proposed in [13]. (c) Frequency dependence, method B (stars, $\eta=0.31$; squares, $\eta=0.22$ ). The measured oscillatory thresholds vary somewhat with the measurement method, but are fairly close to the avalanche case at the lower frequency.

be quite dramatic. These measurements indicate that the concept of well-defined thresholds depending only on acceleration (or angle in the case of avalanches) is simplistic. We propose that the motion allows local compaction to cause the apparent threshold to increase, so that the current $\Gamma$ is then insufficient to allow the motion to continue.

The dependence of the measured thresholds on layer depth is shown in Fig. 4(a). We find that the thresholds measured by methods A,B are slightly different, but both methods show thresholds around $\Gamma=0.45$ for which the dependence on layer depth is quite modest. The depth variation and hysteresis are both smaller for method B.

In Fig. 4(b) we compare the oscillatory flow starting threshold for method A with the avalanche behavior, represented by the tangent of the threshold angle $\tan \left(\theta_{\text {start }}\right)$ for avalanches measured in the same container, in order to minimize any variations due to container size. The dashed curve is a phenomenological fit of the model given in Ref. [13] to

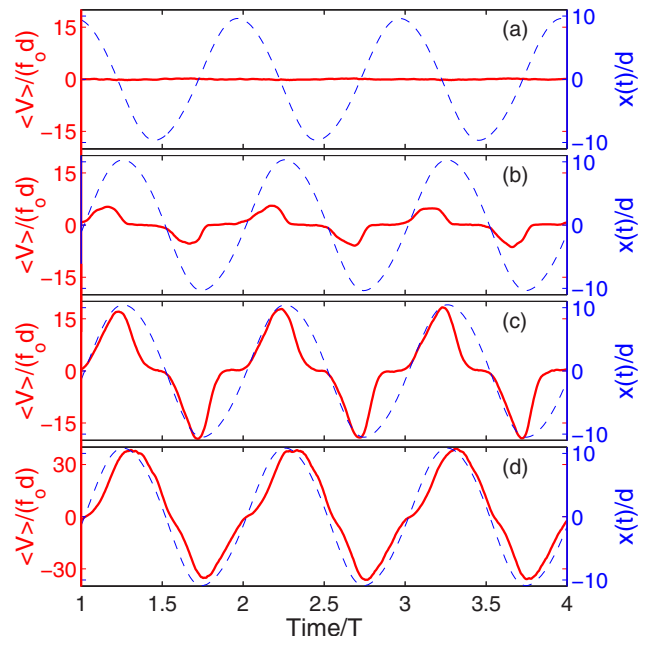

FIG. 5. (Color online) Temporal trace of the spatially averaged $x$ velocity $\langle V\rangle$ along the direction of excitation (full line) at the surface of the layer. Velocities are made nondimensional by $f_{o} d$. The layer depth is $9 d$, and $\eta=0.21$. (a) $\Gamma=0.39$, (b) $\Gamma=0.44$, (c) $\Gamma$ $=0.46$, and (d) $\Gamma=0.48$. Dashed lines show the container position (which is approximately proportional to the effective force). For intermediate accelerations, the rheology changes during the cycle.

our avalanche data. It may be seen that the oscillatory and avalanche experiments are close to each other, though the depth dependence is somewhat weaker for the oscillatory flow.

In Fig. 4(c) we consider the frequency dependence of the measured thresholds, using method B (discarding the first 1 min of data). It can be seen that the apparent thresholds are significantly dependent on the imposed frequency. This fact implies that the dimensionless acceleration is not the only parameter controlling the threshold. Since we are unable to reach the threshold for dimensionless frequencies below $\eta$ $=0.22$, we cannot be certain that the apparent agreement in Fig. 4(b) for the two systems at small $h$ would persist at even lower frequencies. However, the trends with $h$ for the two systems would certainly be similar.

\section{RESULTS: DYNAMICS}

In this section, we consider the details of the motion within the excitation cycle. Figure 5 shows the temporal trace of the average surface $x$ velocity of the tracked particles in the container frame of reference, $\langle V\rangle$, in a layer that is 9 diameters deep (i.e., $h=9 d$ ), at an excitation frequency of $\eta=0.22$ (solid line). The position of the container in the lab frame of reference is also shown, as it is approximately proportional to the effective force acting on the particles. [Note: Small deviations from sinusoidal motion cause the true acceleration and therefore the effective force to differ slightly from the container position curve $x(t)$.] Figure 5(a), where $\Gamma=0.39$ (method B), shows only slight vibrations of surface grains in the box frame. At $\Gamma=0.44$, a clear coherent periodic motion of the grains relative to the box is observed [Fig. 5(b)]. However, the motion is significant over only part of the cycle. As $\Gamma$ is further increased [Fig. 5(c)], the amplitude 


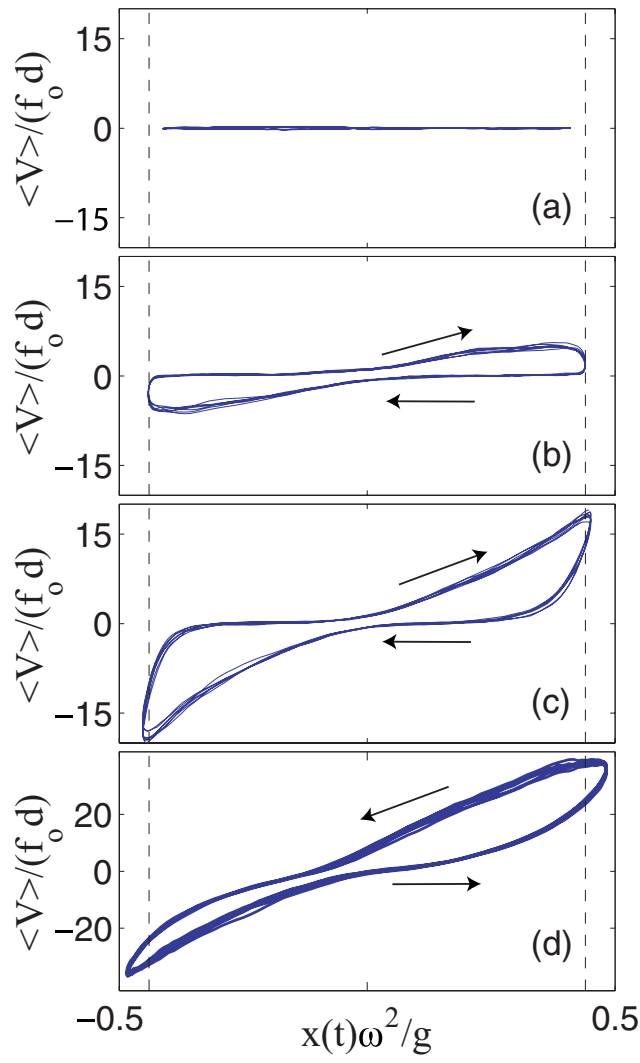

FIG. 6. (Color online) Nondimensional spatially averaged $x$ velocity $\langle V\rangle$ as a function of the approximate instantaneous nondimensional acceleration $x(t) \omega^{2} / g$, for the four accelerations given in Fig. 5. Dashed lines show the stopping thresholds, where $x(t) \omega^{2} / g= \pm \Gamma_{\text {stop. }}$. Arrows show the circulation of the trajectory. In (b) and (c), the material starts to move without threshold, but becomes solid-like as the acceleration falls.

of the motion in the container frame increases, and eventually [Fig. 5(d)] the motion occurs over the entire cycle. The behavior in what we designate as the "intermittent" or partially excited regime is particularly surprising: The velocity in Figs. 5(c) and 5(d) begins to grow when the inertial force is nearly zero (i.e., without a well-defined threshold). In other words, the material behaves as if it is fluidized.

This behavior is revealed more clearly by examining the nondimensional particle velocity (again, in the box frame) as a function of the apparent instantaneous nondimensional acceleration $\Gamma(t)=x(t) \omega^{2} / g$ (which is proportional to the apparent force). This dependence is shown in Fig. 6 for the four cases shown in Fig. 5. Here, the dotted lines show $\Gamma_{\text {stop }}$, so the acceleration is usually below threshold. In Fig. 6(a), the response is weak. In Fig. 6(b) and 6(c) (the intermittent regime), the response velocity varies smoothly with the force, with no threshold. This absence of threshold reflects the fact that the material has been excited in previous cycles. There is substantial hysteresis between the rising and falling parts of the curve. The material becomes nearly solidlike during the decline (i.e., $\langle V\rangle$ is nearly horizontal), not long after the peak acceleration is reached. Furthermore, our vertical profiles discussed later in the paper (Fig. 10) show that the velocity below the surface is always lower than that at the surface, so

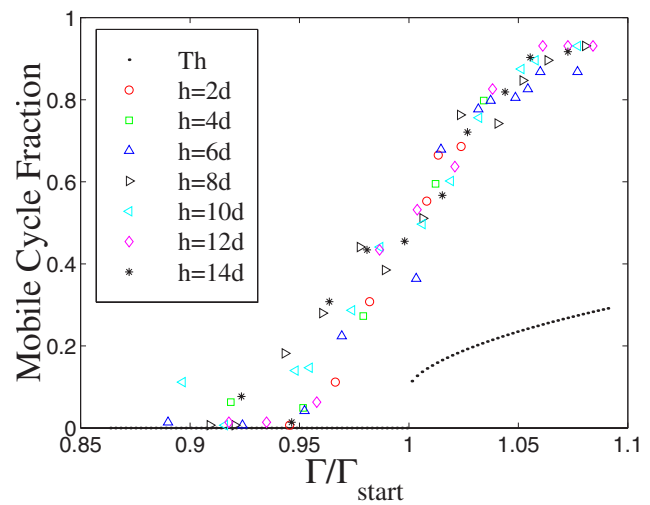

FIG. 7. (Color online) Mobility cycle fraction of the surface layer obtained with method A by decreasing $\Gamma$. The fraction of time for which the surface particles move is plotted as function of the ratio $\Gamma / \Gamma_{\text {start }}$ for seven layer depths. The dotted curve shows the theoretical fraction of time for which $\Gamma$ would be larger than $\Gamma_{\text {stop }}$ once $\Gamma_{\text {start }}$ has been exceeded.

the entire packing must be at rest during this part of the cycle. We think of this as a kind of jamming transition. The fluidization and the jamming observed in different parts of the cycle underline the complex nonlinear rheology of the granular layer.

Finally in Fig. 6(d), where the fluidization is largely complete, jamming appears to be unimportant (the motion persists as acceleration is decreased), but particle inertia may be more important. Notice that (i) the scale of the $y$ axis has been changed for Figs. 5(d) and 6(d), because of the sharp increase of $V_{\text {rms }}$ with $\Gamma$ shown Fig. 2. Also, the phase shift indicated by the arrow is inverted between Fig. 6(c) and Fig. 6(d). In all these cases, the phase difference is far from the value of $\pi / 2$ expected for a simple linear rheology, which would gives an ellipse in Fig. 6. In summary, we find that in the intermittent regime, there is substantial motion at times when $\Gamma<\Gamma_{\text {stop. }}$ These observations indicate that the rheological behavior of the material is not determined by the instantaneous value of $\Gamma$ alone.

The surprising aspects of the behavior of Figs. 5 and 6 are underlined by considering the mobile cycle fraction as a function of the normalized acceleration, as we show in Fig. 7. We define the mobile cycle fraction as the fraction of the time during which the motion is larger than the measurement noise level. Curves are obtained with method A by decreasing $\Gamma$. It may be seen that the mobile cycle fraction grows strongly from very small values to nearly unity over a $10 \%$ range of $\Gamma / \Gamma_{\text {start }}$. If the motion were instantaneously controlled by the thresholds, the mobile cycle fraction would grow starting at $\Gamma / \Gamma_{\text {start }}=1$, and increase only gradually with acceleration. The actual behavior is clearly quite different and much stronger. The measurements imply that the layer is mobile even when $\Gamma_{\text {start }}$ has been exceeded only in the past when $\Gamma$ was larger. A possibly similar memory effect has been found in a granular Couette cell where the motion depends on the history of the previous direction of the applied shear [22].

Further insight can be obtained by examining the time dependence of the velocity fluctuations, characterized by 


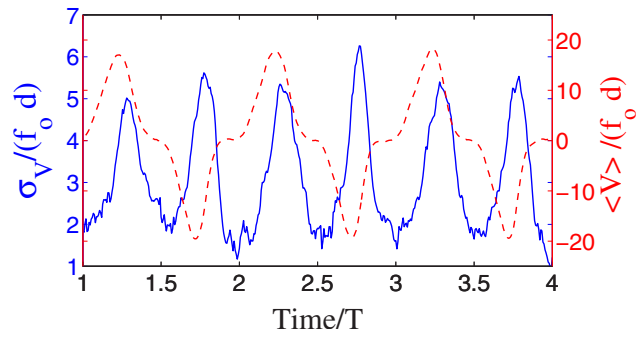

FIG. 8. (Color online) Time dependence of the velocity standard deviation $\sigma_{V}$ (including both components), compared to the mean particle velocity (dashed). The fluctuations are largest close to (but slightly later than) the instant of the maximum velocity along the direction of excitation.

their standard deviation $\sigma_{V}$ (including both horizontal directions). This quantity is presented in Fig. 8 for the data shown in Fig. 5(c), and compared to the velocity. At each instant of time, spatial averages are presented. It is interesting to note that the fluctuation reaches its peak slightly after the velocity itself does. This behavior presumably is a consequence of the fact that the layer is never at a steady state.

\section{RESULTS: VELOCITY PROFILES}

We also determined the instantaneous velocity profiles across the cell along $y$ at the free surface, and also vertically along $z$ near the side wall. The shapes of these profiles turn out to be time-dependent in the oscillating flow. The profiles reported here were made in the center of the cell along the $x$ direction, where the velocity amplitude is nearly independent of $x$. The amplitude declines smoothly to zero at the endwalls.

The time evolution of the velocity viewed from the top for different distances to the sidewall, $V_{y}(t)$, is shown by the full lines in Fig. 9, at $h=9 d$ for $\Gamma=0.45$ (in the intermittent regime) and for $\Gamma=0.49$, where the flow is fully mobilized. The (red) dashed line shows the spatially averaged instantaneous velocity. The insets present some instantaneous velocity profiles, $V_{t}(y)$, normalized by the spatial average at the corresponding time $\langle V\rangle_{t}$. The intermittent regime is shown in Fig. 9(a) $(\Gamma=0.45)$. Particles near the wall are almost at rest during the entire oscillation cycle, and particles in the middle of the cell move during part of the cycle. In this measurement (which is made over half of the cell width), the motion is time-asymmetric between positive and negative velocity. (However, the asymmetry varies from run to run, depending perhaps on the preparation of the layer.) The particles near the wall reach their maximum amplitude before the particles in the center do, and their velocities fall before those of particles in the center.

This behavior appears more clearly in the velocity profiles shown in the inset, which reveal different behavior during the rising and falling of the velocity magnitude. During the rising part, the velocity increases smoothly from the wall to center with a finite linear gradient at the wall (red asterisks) whereas during the falling part (green crosses), there is a fairly sharp transition between the particles at rest near the wall and those that are flowing near the center. The fact that
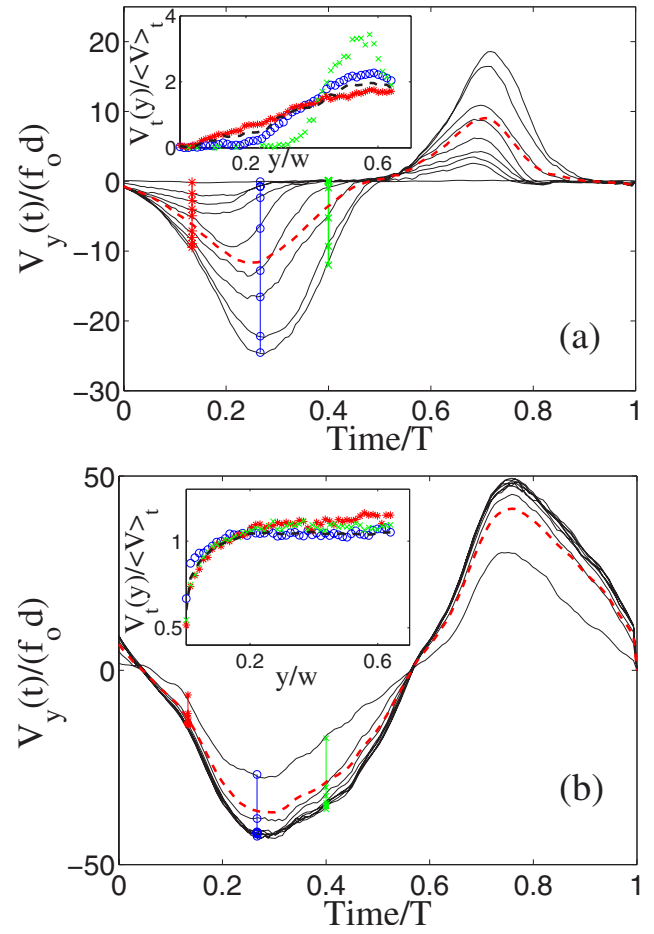

FIG. 9. (Color online) Temporal traces of the $x$ velocities at the surface for different distances to the sidewall, $V_{y}(t)$ (full lines). (a) $\Gamma=0.45$ (intermittent regime) and (b) $\Gamma=0.49$ (fully mobilized at the top). In both cases $h=9 d$ and $\eta=0.21$. The distance between two successive curves is about $2 d$. The dashed line is the spatially averaged velocity $\langle V\rangle_{t}$. The vertical cuts through these curves show the instantaneous velocity profiles $V_{t}(y)$ at different times, represented in the inset by the same symbols. The profiles are normalized by the spatially averaged velocity at the corresponding time. The dashed line in the inset is the profile of the rms (time-averaged) surface velocity normalized by its space averaged value. The crosscell profile of the horizontal velocity is clearly time-dependent in the intermittent regime.

these profiles have a time-dependent shape implies a clear difference between these oscillatory flows and steady avalanche flows. Even the rms (time-averaged) surface velocity normalized by its space averaged value (dashed line) does not agree with the numerical prediction based on the constitutive law proposed in [14], which works well for steady flows. However, it is known that this constitutive law fails to predict avalanche flows close to the flow threshold, where large structures and long range correlations are present [11].

In the fully developed regime $(\Gamma=0.49)$, shown in Fig. 9(b), even the particles near the sidewall move during the entire cycle. Farther from the wall than a boundary layer of about $10 d$, the velocities saturate to a value almost equal to the space averaged velocity of the surface. This is more clearly seen in the inset. The resulting instantaneous profile as well as the normalized rms profile qualitatively agrees with the prediction [14] for low flow rate. However a more quantitative comparison would necessitate some knowledge of the flow below the surface so that the flux could be determined.

Although Fig. 9 shows that the wall friction induces a boundary layer so that the velocity close to the sidewall falls 

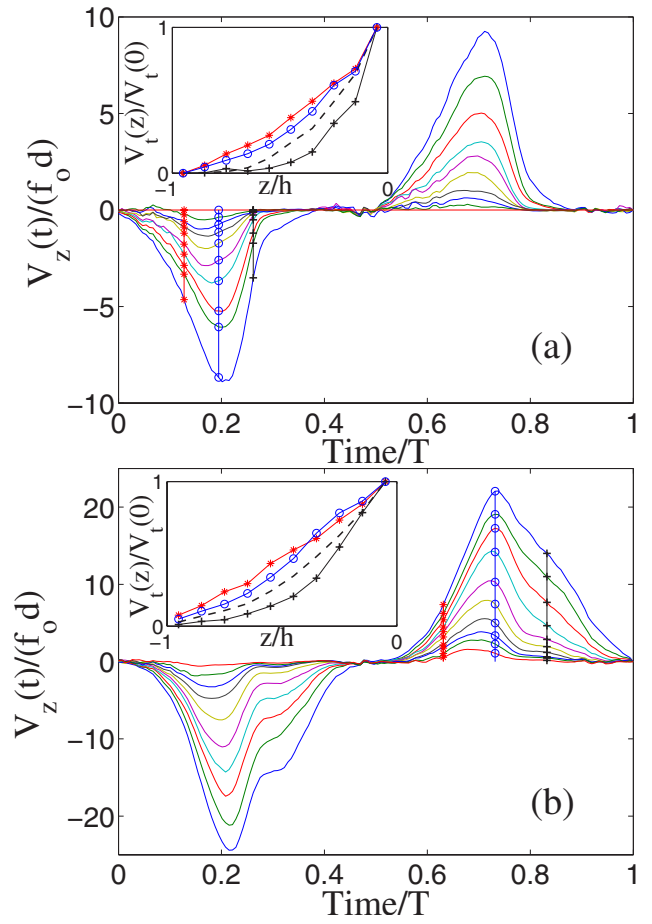

FIG. 10. (Color online) Temporal traces of the $x$ velocity at the sidewall $V_{z}(t)$ (full lines) for different distances below the surface. (a) $\Gamma=0.45$ (intermittent regime) and (b) $\Gamma=0.49$ (fully mobilized at the top). In both cases $h=9 d$ and $\eta=0.21$. The vertical separation between two successive curves is about one particle diameter. Vertical cuts through these curves show the instantaneous velocity profiles $V_{t}(z)$ at different times, represented in the inset by the same symbols. The profiles are normalized by the velocity of the top layer. The dashed line in the inset is the profile of the rms (timeaveraged) surface velocity normalized by the rms surface velocity. The vertical profile of the horizontal velocity is clearly time-dependent.

below the velocity in the bulk, we are able to characterize the depth dependence within this boundary layer, as a function of time. Moreover, due to the opacity of dry granular material, this is the usual way to explore the depth dependence in avalanche experiments as well [24]. The measurements, realized under almost same conditions as those made from the top, are presented in Fig. 10. They have several features in common with the top measurements. Figure 10(a), with $\Gamma$ slightly above 0.45 , shows that the intermittent regime is not just a surface phenomenon but it implies that motion occurs during a part of the cycle deep within the layer. Similarly to the top measurements, the vertical dependence also reveals an asymmetry between rising and falling velocity magnitudes. The inset shows that during the rising part (asterisks), the instantaneous velocity, normalized by the velocity of the top layer at the same time, increases smoothly from the bottom to the top of the layer. During the falling part (crosses), on the other hand, the flow is largely confined to the upper part of the layer. In contrast to the flow profiles measured from the top, the flow localization noticed in the intermittent regime is also observable in the full developed regime shown in Fig. 10(b). However, we cannot tell if this behavior is also true in the bulk, far from the sidewalls.
Far from the sidewalls, the boundary condition on the upper surface of the layer is zero stress. Many rheologies predict in this case a velocity profile of the form

$$
V(z) / V_{o}=\left[1-|z / h|^{\delta}\right],
$$

where $\delta=2$ is the Newtonian case and $\delta=3 / 2$ comes from any theory that introduces no new dimensional parameters or fields. Equation (2) implies that $d V / d z$ is always zero at the upper surface. Some sidewall measurements in $2 \mathrm{D}$ and $3 \mathrm{D}$ avalanche experiments show velocity profiles in agreement with Equation (2) [24-26]. When it is assumed to apply to time-periodic flows, it implies that the derivative of the rms average velocity must also vanish at $z=0$. The insets of Fig. 10 show that this is not the case. For both values of $\Gamma$ that we have explored in detail, neither the instantaneous vertical profile nor the profile of the rms velocity can be fitted by Eq. (2) because $d V / d z$ does not vanish at the upper surface.

We cannot exclude the possibility that sidewall friction affects our results. It could possibly explain the nonvanishing slope at the free surface that we observe in contrast to what Eq. (2) would imply [14]. Moreover, many experiments and simulations have observed almost linear velocity profiles $(\delta$ $=1$ ) for shallow layer steady flows $[4,13]$. This roughly linear behavior is observed in our experiment only during the rising phase of the velocity amplitude. It seems likely that the profiles seen here reflect the complex nonlinear rheology characteristic of time-dependent experiments.

\section{DISCUSSION AND CONCLUSIONS}

At very low forcing frequency (but constant acceleration), our experiments on oscillatory forcing of a horizontal granular layer should correspond to the threshold behavior of a tilted layer. However, at finite frequency and beyond the threshold, this analogy breaks down because there are actually multiple time scales. Our ability to reach the low frequency behavior is limited by the large amplitude $A$ that would be required.

There are three physical time scales in the oscillatory system. The first is given by the driving frequency $T=2 \pi / \omega$, while the second is the local particle rearrangement time $T_{2}=\sqrt{d / g}$, where $d$ is the particle diameter and $g$ is the effective gravity. We have written the ratio of these time scales as $\eta=\omega \sqrt{d / g}=2 \pi T_{2} / T$. Finally there is the timescale for the flow to reach the steady state, where this is possible without jamming. This timescale is roughly $T_{3}=T \sqrt{h / A}=\sqrt{h / g}$, where $h$ is the layer depth and $A$ the driving amplitude, if we reason by analogy to avalanche flows. We can alternately write this third time scale as $T_{3}=T_{2} \sqrt{h / d}$. Thus, there is one primary frequency $\eta$ and there are two natural length ratios $h / d$ and $h / A$. The first of these is the depth in particle diameters, which we have analysed in detail. The second group $h / A$ determines whether or not flows can approach a steady state velocity. For small values of this ratio, a steady state would be expected (far above threshold to avoid jamming). However, we are not in this limit except for the shallowest layers. When this parameter is sufficiently large, shear waves should propogate into the layer and decay. This would also be interesting to study, but was not our focus. 
Thresholds. We began our study by comparing the threshold behavior of granular flow for oscillatory excitation with the corresponding behavior for avalanches of inclined layers, using the same container to minimize changes in the boundary effects. We note that the measured thresholds depend significantly on the measurement protocol, as a result of reorganization of the material over a few tens of seconds that appears to raise the threshold. While transient motion also occurs in avalanches, the motion here appears to be global.

Therefore, the functional form of $V_{\mathrm{rms}}(\Gamma)$ depends on whether the material has been allowed to evolve or not, as does the difference between $\Gamma_{\text {start }}$ and $\Gamma_{\text {stop }}$. However, the depth dependence of the thresholds is only weakly dependent on the protocol. We compare the behavior of $\Gamma_{\text {start }}$ and $\Gamma_{\text {stop }}$ for the oscillatory flow to the angular threshold $\tan \left(\theta_{\text {start }}\right)$ for the inclined flow in the same container (Fig. 3). The two systems behave similarly, but the depth dependence is slightly stronger in the latter case. There is significant frequency dependence, with the thresholds rising significantly as $\eta$ is increased beyond $0.25\left(f_{o}=3 \mathrm{~Hz}\right)$, the lowest frequency we could reach. We expect that the thresholds will increase indefinitely with $\eta$ at fixed $\Gamma$ since oscillations with amplitudes smaller than the grain diameter will not propagate into the bulk.

Our experiments agree with others [13] in which the lower boundary is rough and the thresholds decline with increasing depth. Preliminary measurements with a smooth plexiglass bottom show a decrease of $\Gamma_{\text {start }}$ to around 0.31 for $\eta=0.21$ (and 0.32 for $\eta=0.31$ ), and $\tan \left(\theta_{\text {start }}\right)=0.34$ for the avalanche experiment. We find no dependence on the layer depth in this case. These values of $\Gamma_{\text {start }}$ are smaller than the ones reported in [21], where an increase of $\Gamma_{\text {start }}$ with $h$ is also observed. This contradictory observation is probably due to the narrowness of the channel used in that case, and the resultant larger role of sidewall friction. In our experiments, stresses from the vibration are transmitted primarily through the base of the cell.

Dynamics. Our observations of the time-dependent dynamics of the flowing grains highlight significant differences between the oscillatory and avalanche experiments. The dynamics during the flow cycle (Figs. 5 and 6) is especially surprising, in that for accelerations close to the flow threshold, the material can be fluid-like during part of the cycle, and solid-like during the remainder. Jamming can occur near the peak acceleration, and its origin is not yet understood. It would be desirable to investigate this behavior at frequencies below those reported here, but this is impractical because much larger amplitudes would be needed to reach the critical acceleration. We expect that the flow could be confined to a smaller fraction of the (longer) cycle at lower frequencies, because the time required to jam is presumably given by the ratio of the container length to the velocity relative to the container, and the latter is determined by the applied acceleration.

It is interesting to note that flow can occur even if the static threshold $\Gamma_{\text {start }}$ is not currently being exceeded at any time during the cycle, provided that $\Gamma_{\text {stop }}$ is exceeded at some point during the cycle. Fluidization is not determined by the current acceleration. Once motion begins, it can persist and even build up over the cycle, even if $\Gamma$ is currently below $\Gamma_{\text {stop. }}$. As a result, the mobile cycle fraction rises far faster with acceleration than the static thresholds would lead one to expect (Fig. 7).

We find that the velocity fluctuations (Fig. 8) reach their largest values slightly later than the instant of largest mean velocity. This presumably reflects the fact that we are far from the steady state.

The flow profile across the top of the cell (along $y$, perpendicular to the direction of flow), and the vertical profile along $z$ in the boundary layer (Figs. 9 and 10) reveal interesting dynamics. The most important observation is that these profiles are time-dependent, in contrast to the avalanche flow. Furthermore, the behavior during the rising and falling parts of the velocity cycle is quite different. In the intermittent regime, when the velocity is increasing, the gradients along $y$ and $z$ are roughly linear, while when the velocity is decreasing, flow ceases earlier near the sidewall (seen from the top) and far below the surface (seen from the side). When the flow is fully excited during the entire cycle, the profile along $y$ is qualitatively similar to that seen in avalanche flows. However, in the intermittent regime, the rheology is strongly nonlinear. As we remarked in Sec. V, more complex behavior also occurs near threshold for avalanche flows, but the current experiment with periodic excitation allows a detailed study, as the effective driving forces are small during part of each cycle.

In spite of its simplicity, a horizontally oscillated granular layer shows rich dynamics. Just as ac shear can reveal the complex rheology of materials, it can be a useful method of exploring the behavior of granular materials. Several features of the work presented here could be useful tests of theoretical or numerical modeling, including the transient decay of the oscillation amplitude just above threshold (Fig. 3), the timedependent mean velocity of Fig. 6, and the time-dependent velocity profile shown in Fig. 9. In a second paper, we will present numerical computations that address some of these issues [27]. One important omission from the current experimental work is to explore the phenomena as a function of material properties and boundary conditions. We have made a few preliminary observations, but this will require a major investigation [28].

\section{ACKNOWLEDGMENTS}

This work was supported by the National Science Foundation, Division of Materials Research, under Grant No. DMR-0405187. It was started while J.N.M. and J.P.G. were visitors at the Kavli Institute for Theoretical Physics at U.C. Santa Barbara. J.N.M is supported by the UK Engineering and Physical Sciences Research Council (GR/TO2416101). 
[1] A. Daerr and S. Douady, Nature (London) 399, 241 (1999).

[2] M. Y. Louge and S. C. Keast, Phys. Fluids 13, 1213 (2001).

[3] O. Pouliquen and N. Renaut, J. Phys. II 6, 923 (1996).

[4] L. Silbert et al., Phys. Rev. E 64, 051302 (2001).

[5] L. Silbert, J. W. Landry, and G. S. Grest, Phys. Fluids 15, 1 (2003).

[6] S. B. Savage and K. Hutter, J. Fluid Mech. 199, 177 (1989).

[7] J.-P. Bouchaud et al., J. Phys. I 4, 1383 (1994).

[8] I. S. Aranson and L. S. Tsimring, Phys. Rev. E 64, 020301 (2001).

[9] A. Aradian, E. Raphael, and P. G. de Gennes, Phys. Rev. E 60, 2009 (1999).

[10] B. Andreotti, A. Daerr, and S. Douady, Phys. Fluids 14, 415 (2002).

[11] O. Pouliquen et al., in Powders and Grains, edited by R. Garcia-Rojo, H. Hermann, and S. McNamara (Taylor \& Francis Group, Stuttgart, 2005), Vol. 2, p. 859.

[12] O. Pouliquen, Phys. Fluids 11, 542 (1999).

[13] GDR MiDi, Eur. Phys. J. E 14, 341 (2004).

[14] P. Jop, Y. Forterre, and O. Pouliquen, Nature (London) 441, 727 (2006).

[15] P. Jop, Y. Forterre, and O. Pouliquen, J. Fluid Mech. 486, 21 (2005).
[16] G. H. Ristow, G. Strassbruger, and I. Rehberg, Phys. Rev. Lett. 79, 833 (1997).

[17] G. Strassbruger and I. Rehberg, Phys. Rev. E 62, 2517 (2000).

[18] P. M. Reis and T. Mullin, Phys. Rev. Lett. 89, 244301 (2002).

[19] S. G. K. Tennakoon, L. Kondic, and R. P. Behringer, Europhys. Lett. 45, 470 (1999).

[20] M. Medved, H. M. Jaeger, and S. R. Nagel, Europhys. Lett. 52, 66 (2000).

[21] G. Metcalfe et al., Phys. Rev. E 65, 031302 (2002).

[22] M. Toiya, T. Stambaugh, and W. Losert, Phys. Rev. Lett. 93, 088001 (2004)

[23] The particle tracking routines were developed by J. Crocker and E. Weeks. See http://www.physics.emory.edu/ weeks/idl/.

[24] S. B. Savage, J. Fluid Mech. 92, 53 (1979).

[25] E. Azanza, F. Chevoir, and P. Moucheront, J. Fluid Mech. 400, 199 (1999).

[26] C. Ancey, Phys. Rev. E 65, 011304 (2001).

[27] J. N. McElwaine et al. (unpublished).

[28] Experiments with rough aluminum grains (and a rough bottom surface) show a large increase of $\Gamma_{\text {start }}$ (by approximately $25 \%$ at $\eta=0.22)$, but $\tan \left(\theta_{\text {start }}\right)$ is even more strongly affected, with an increase of $58 \%$. 